\title{
Colorimetric determination of urinary adenosine using aptamer-modified gold nanoparticles
}

\author{
Shih-Ju Chen, Yu-Fen Huang, Chih-Ching Huang, Kun-Hong Lee, \\ Zong-Hong Lin, Huan-Tsung Chang* \\ Department of Chemistry, National Taiwan University, 1, Section 4, \\ Roosevelt Road, Taipei 106, Taiwan
}

Received 19 October 2007; received in revised form 3 January 2008; accepted 6 February 2008

Available online 16 February 2008

\begin{abstract}
This paper describes a colorimetric sensing approach for the determination of adenosine triphosphate (ATP) using aptamer-modified gold nanoparticles (Apt-Au NPs). In the absence of the analytes, the color of the Apt-Au NPs solution changed from wine-red to purple as a result of salt-induced aggregation. Binding of the analytes to the Apt-Au NPs induced folding of the aptamers on the Au NP surfaces into four-stranded tetraplex structures (G-quartet) and/or an increase in charge density. As a result, the Apt-Au NPs solution was wine-red in color in the presence of the analytes under high salt conditions. For mixtures of ATP (20.0-100.0 nM), Apt-Au NPs (3.0 nM), 10.0\% poly(ethylene glycol), $0.2 \mu M$ TOTO-3, $150.0 \mathrm{mM} \mathrm{NaCl}, 15.0 \mathrm{mM} \mathrm{KCl}$, and $16.0 \mathrm{mM}$ Tris- $\mathrm{HCl}(\mathrm{pH} 7.4)$, a linear correlation $\left(R^{2}=0.99\right)$ existed between the ratio of the extinctions of the Apt-Au NPs at 650 and $520 \mathrm{~nm}\left(E x_{650 / 520}\right)$ and the concentration of ATP. The limit of detection for ATP was $10.0 \mathrm{nM}$. The practicality of this simple, sensitive, specific, and cost-effective approach was demonstrated through the determination of the concentration of adenosine in urine samples.
\end{abstract}

(C) 2008 Elsevier B.V. All rights reserved.

Keywords: G-quartet; Aptamer; Gold nanoparticle; Sensor; Adenosine triphosphate (ATP); Urine sample

\section{Introduction}

Gold nanoparticles (Au NPs) have emerged as important colorimetric reporters because of their high extinction coefficients and strongly distance-dependent optical properties (Kim et al., 2001; Huang et al., 2005). Aggregation-based $\mathrm{Au}$ NP nanosensors have been utilized to investigate several types of interactions, including those between two DNA molecules (Elghanian et al., 1997), proteins and ligands (Nam et al., 2002), and antigens and antibodies (Shenton et al., 1999).

Aptamers are DNA or RNA sequences created in vitro through systematic evolution of ligands by exponential enrichment (SELEX) for the recognition of target analytes with high affinity and specificity (Tuerk and Gold, 1990). Aptamerfunctionalized $\mathrm{Au}$ NPs have been applied to the analysis of

\footnotetext{
* Corresponding author. Tel.: +886 233661171 ; fax: +886 233661171.

E-mail address: changht@ntu.edu.tw (H.-T. Chang).
}

several analytes, including proteins, metal ions, and small organic compounds (Wang et al., 2006; Li et al., 2007; Wu et al., 2007).

Adenosine triphosphate (ATP) plays important roles in regulating cellular metabolism and biochemical pathways (Pérez-Ruiz et al., 2003). Several strategies have been reported to the detection of ATP, including aptamer-based optical sensing, capillary electrophoresis, mass spectrometry, enzymatic assays, and electrochemical biosensors (Jhaveri et al., 2000; Michaud et al., 2004; Yoshida et al., 2006; Huang and Chang, 2007; Lai et al., 2007). Colorimetric assays using Au NPs were developed for determining ATP (Liu and Lu, 2006; Zhao et al., 2007). Our goal in this study was using aptamer-modified Au NPs (Apt-Au NPs) as a sensitive colorimetric sensor for ATP. The aptamer (Huizenga and Szostak, 1995) we used is one that binds two ATP molecules in a non-canonical, but stable, helix comprising G:G and G:A base pairs flanked by short canonical helices (Lin and Patel, 1997). We evaluated the effects of several parameters - including the ionic strength, the aptamer density on the Au NPs, the concentration of TOTO-3, and the 
presence of additives such as poly(ethylene glycol) (PEG) - on the sensitivity of this ATP assay.

\section{Experimental section}

\subsection{Chemicals}

Sodium tetrachloroaurate(III) dehydrate, 6-mercaptohexal1-ol (6-MH), adenosine, ATP, adenosine monophosphate (AMP), adenosine diphosphate (ADP), guanosine triphosphate (GTP), cytidine triphosphate (CTP), and uridine triphosphate (UTP) were obtained from Sigma (St. Louis, MO). Citric acid, trisodium citrate, and PEG $\left(M_{\mathrm{W}}=2000 \mathrm{Da}\right)$ were purchased from Aldrich (Milwaukee, WI). Sodium chloride and potassium chloride were obtained from Acros (Geel, Belgium). TOTO3 and YOYO-3 were purchased from Molecular Probes, Inc. (Eugene, OR). Ethidium bromide (EtBr) was purchased from Amersham Biosciences (Japan). The 5'-thiol-modified aptamer having the sequence $5^{\prime}$-TTT TTT ACC TGG GGG AGT ATT GCG GAG GAA GGT-3' was purchased from Integrated DNA Technologies, Inc. (Coralville, IA).

\subsection{Synthesis of Au NPs and Apt-Au NPs}

An aqueous solution of $38.8 \mathrm{mM}$ trisodium citrate solution ( $25.0 \mathrm{~mL}$ ) was rapidly added to $250.0 \mathrm{~mL}$ boiling aqueous solution containing $1.0 \mathrm{mM} \mathrm{NaAuCl}_{4}$, and stirred for $15 \mathrm{~min}$. The sizes of the Au NPs were verified through transmission electron microscopy (TEM) using a Hitachi H7100 TEM instrument (Tokyo, Japan) operated at $75 \mathrm{kV}$; with an average size of $13.3 \pm 1.2 \mathrm{~nm}$ (from 1000 counts). A GBC Cintra 10e doublebeam UV-vis spectrophotometer (Victoria, Australia) was used to measure the absorbance of the Au NPs in citrate solutions.
An aliquot of the aqueous Au NP solution $(988.0 \mu \mathrm{L})$ was mixed with the thiol-modified 33-mer DNA oligonucleotide $(100.0 \mu \mathrm{M}, 12.0 \mu \mathrm{L})$ at room temperature for $12 \mathrm{~h}$ (Storhoff et al., 1998). The mixtures were then centrifuged for $15 \mathrm{~min}$ at $16,000 \mathrm{rpm}$ to remove the excess thiol-oligonucleotides. After two centrifuge/wash cycles, the colloids were resuspended in $4.0 \mathrm{mM}$ trisodium citrate $(1.0 \mathrm{~mL})$. The concentration of displaced oligonucleotide molecules by 2-mercaptoethanol was determined through the addition of OliGreen (ssDNA reagent, Molecular Probe, Eugene, OR) and then measuring the fluorescence intensities at $524 \mathrm{~nm}$ (excitation wavelength: $480 \mathrm{~nm}$ ). The results indicated that ca. 70 oligonucleotide molecules were attached to each Au NP.

\subsection{Apt-Au NP sensing assays}

Mixtures $(90.0 \mu \mathrm{L})$ of probe solutions and ATP $(0-1.2 \mu \mathrm{M})$ were placed in 96-well plates at room temperature for $30 \mathrm{~min}$. The probe solution contained $16.0 \mathrm{mM}$ Tris- $\mathrm{HCl}(\mathrm{pH}$ 7.4), $15.0 \mathrm{mM} \mathrm{KCl}$, Apt-Au NPs (3.0 nM), 10.0\% PEG, and $0.2 \mu \mathrm{M}$ TOTO-3 Aliquots of $10.0 \mu \mathrm{L} \mathrm{NaCl}(1.5 \mathrm{M})$ were added to the mixtures and then equilibrated for another 10 min prior to UV-vis absorption measurements using a $\mu$ Quant Biotek Instruments microplate reader (Winooski, VT, USA).

\subsection{Preparation of urine samples}

Freshly voided urine samples were obtained from four healthy volunteers. Each sample was filtered through a $0.2-\mathrm{mm}$ membrane to remove particulate matter. The human-urine samples $(1.0 \mu \mathrm{L})$ were diluted separately by a factor of 100 with the probe solution and then were equilibrated at $25^{\circ} \mathrm{C}$ for $30 \mathrm{~min}$.
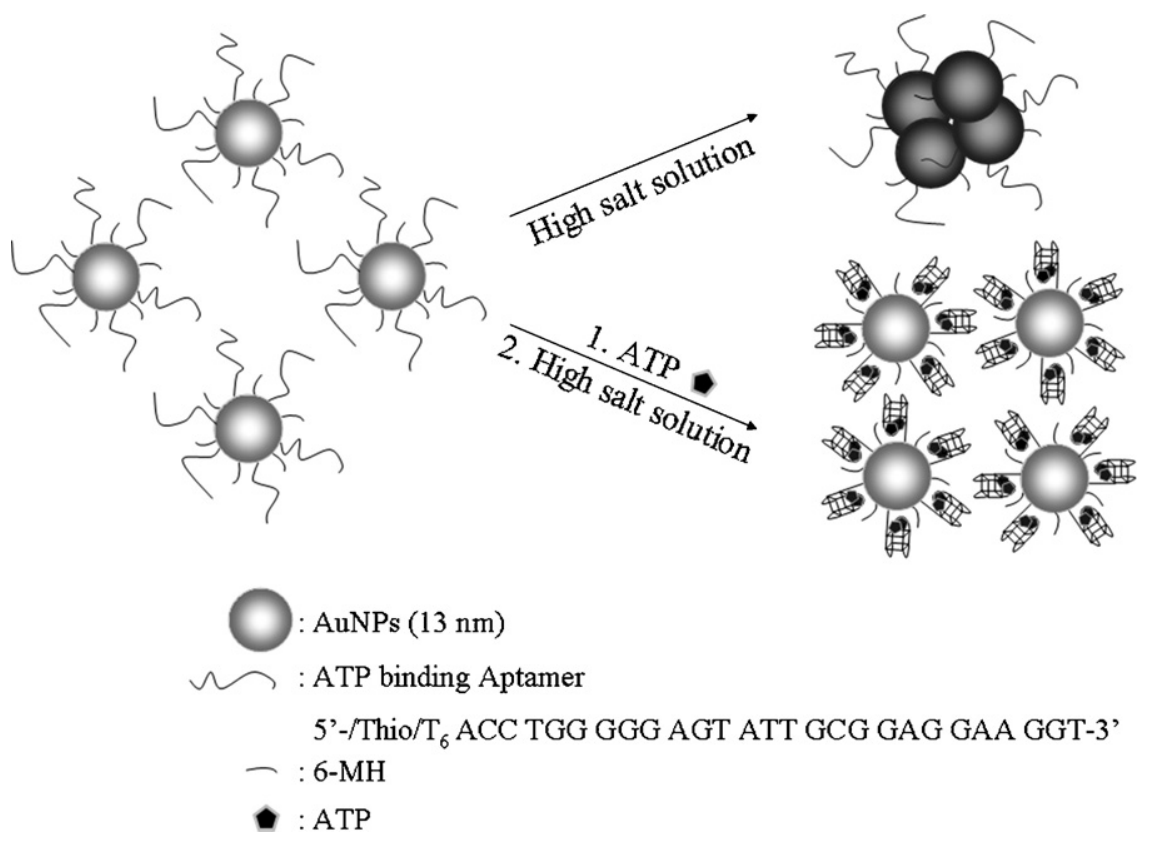

Scheme 1. Schematic representation of the sensing mechanism of Apt-Au NPs for the colorimetric determination of ATP. 
High salt $(150.0 \mathrm{mM} \mathrm{NaCl})$ was then added into the resulting solutions for another $10 \mathrm{~min}$ before measurements.

\section{Results and discussion}

\subsection{Sensing mechanism}

Scheme 1 displays the sensing mechanism of the Apt-Au NPs for colorimetric determination of adenosine and its phosphorylated derivatives (ATP as a representative). Aiquots of salt (e.g. $300.0 \mathrm{mM} \mathrm{NaCl}$ ) are added to Apt-Au NPs in solutions with/without containing ATP. The solution without containing ATP changes color from wine-red to purple as a result of aggregation, while one containing ATP retaine its original red-wine color. A decreased zeta potential due to salt screening is the main cause of instability of the Apt-Au NPs solutions without ATP. Once ATP bound to the Apt-Au NPs, the aptamer on the $\mathrm{Au}$ NP surfaces folded into a four-stranded tetraplex structure (G-quartet) (Wang et al., 2005). In addition, increased negative charge density on the Apt-Au NP surfaces occurs. As a result, the ATP-Apt-Au NPs are stable and retain their wine-red color under high salt conditions.

Fig. 1A displays the UV-vis absorption responses of $3.0 \mathrm{nM}$ Apt-Au NPs in $16.0 \mathrm{mM}$ Tris- $\mathrm{HCl}$ solutions ( $\mathrm{pH}$ 7.4) containing $15.0 \mathrm{mM} \mathrm{KCl}$ and $300.0 \mathrm{mM} \mathrm{NaCl}$ in the absence and presence of $10.0 \mu \mathrm{M}$ ATP. In the presence of ATP, the dispersed Apt-Au NPs displayed an extinction band (surface plasmon resonance band) at $520 \mathrm{~nm}$ (curve b). In contrast, the Apt-Au NPs aggregated in the absence of ATP (curve a). The extinction coefficients at 650 and $520 \mathrm{~nm}$ are related to the quantities of dispersed and aggregated Apt-Au NPs, respectively. Thus, we used Ex 650/520, the ratio of the extinction values at these two wavelengths, to express the molar ratio of the aggregated and dispersed Apt-Au
NPs. The inset to Fig. 1A displays the purple and wine-red colors of the solutions in the absence and presence of ATP, respectively. Fig. 1B and C display TEM and optical dark-field scattering (DFS) images of the Apt-Au NP solutions, respectively, in the absence and presence of ATP. The images in Fig. 1B(a) and $\mathrm{C}$ (a) provide strong evidence for the aggregation of the Apt-Au NPs under high salt conditions in the absence of ATP. Because of the low scattering intensity and faster diffusion of the 13.3$\mathrm{nm} \mathrm{Au}$ NPs, the scattering light from single Apt-Au NPs was hardly observed by our DFS system (Fig. 1C(b)). Strong scattering occurred once the Apt-Au NPs had aggregated; the orange and red spots correspond to scattering images of Apt-Au NP aggregates.

\subsection{Impact of salt and aptamer density on the stability of Apt-Au NPs}

Monitoring the extinction ratio $E x_{650 / 520}$ allowed us to investigate the effect of salt $(0-400.0 \mathrm{mM})$ on the stability of Apt-Au NP solutions containing 20, 35, and 70 surface aptamers per $\mathrm{Au}$ NP (Fig. S1). Extinction ratios indicates degrees of aggregation; high values show great aggregation. For simplicity, we use the descriptors 20-, 35-, and 70-Apt-Au NPs to represent the nanoparticles in these three Apt-Au NP solutions. As exhibited in Fig. S1A, the 20-, 35-, and 70-Apt-Au NPs aggregated at $\mathrm{NaCl}$ concentrations greater than 200.0,250.0, and $350.0 \mathrm{mM}$, respectively. Of the three solutions, the 70-Apt-Au NPs could tolerate the most salt, as expected. The lower $E x_{650 / 520}$ values indicate that the three Apt-Au NP solutions containing ATP are more stable than those without containing ATP (Fig. S1B). Fig. S1C depicts the results obtained after subtracting the values of the extinction ratios $E x_{650 / 520}$ in Fig. S1B from those in Fig. S1A under the same conditions (i.e., the same salt and aptamer den-
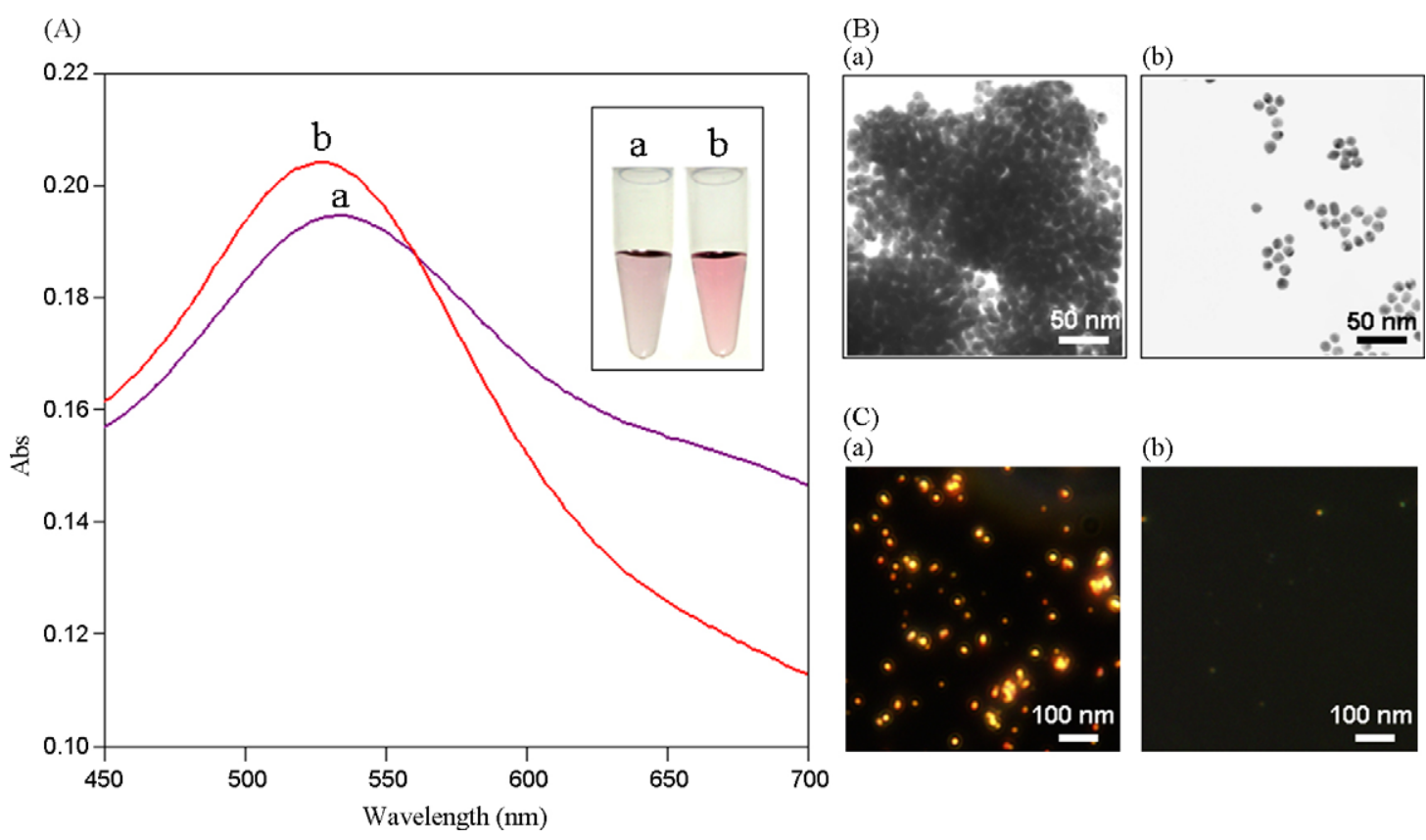

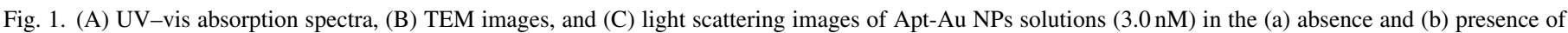

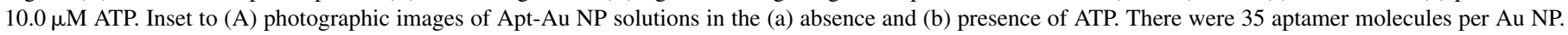
Buffer: $16.0 \mathrm{mM}$ Tris- $\mathrm{HCl}(\mathrm{pH} 7.4)$ containing $15.0 \mathrm{mM} \mathrm{KCl}$ and $300.0 \mathrm{mM} \mathrm{NaCl}$. The reaction time was $30 \mathrm{~min}$. 


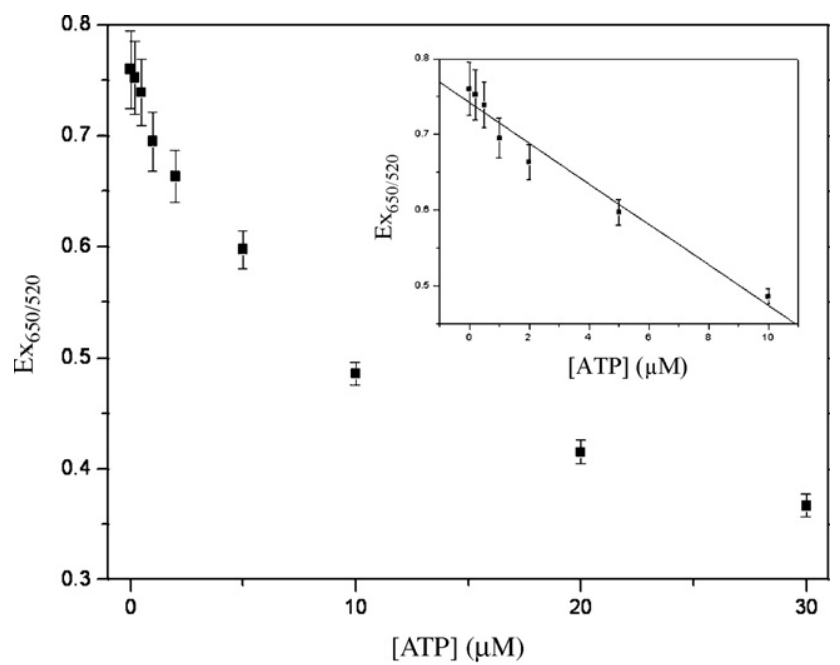

Fig. 2. Responses in the values of $E x_{650 / 520}$ of 35-Apt-Au NP solutions after the addition of ATP at concentrations of 0, 0.2, 0.5, 1.0, 2.0, 5.0, 10.0, 20.0, and $30.0 \mu \mathrm{M}$. The inset indicates that the curve was linear over the range $0.2-10.0 \mu \mathrm{M}$ ATP. Other conditions were the same as those described in Fig. 1.

sities). The largest value of $\Delta E x_{650 / 520}$ suggested that solutions containing 35-Apt-Au NPs and $300.0 \mathrm{mM} \mathrm{NaCl}$ are optimal for detecting ATP.

Next, we used the 35-Apt-Au NPs to determine ATP in solutions consisting of $300.0 \mathrm{mM} \mathrm{NaCl}, 15.0 \mathrm{mM} \mathrm{KCl}$, and $16.0 \mathrm{mM}$ Tris- $\mathrm{HCl}$ (pH 7.4). As exhibited in Fig. 2, the 35-Apt-Au NPs responded to ATP at concentrations over the range $0-30.0 \mu \mathrm{M}$. Upon increasing the concentration of ATP, the value of the ratio $E x_{650 / 520}$ decreased. A linear correlation existed between the value of $E x_{650 / 520}$ and the concentration of ATP over the range $0.2-10.0 \mu \mathrm{M}\left(R^{2}=0.97\right)$. The LOD for ATP was $0.1 \mu \mathrm{M}$ $(\mathrm{S} / \mathrm{N}=3)$.

\subsection{Sensitivity improvement}

To demonstrate changes in the aptamers' conformations, we added DNA intercalators to the 35-Apt-Au NP solutions. The aptamer possesses two stacked G-quartets and two short double-helix stems (Huizenga and Szostak, 1995; Wang et al., $2005)$. The value of $\Delta E x_{650 / 520}$ of the 35-Apt-Au NPs in the presence of ATP decreased to a greater extent when intercalators were present. Fig. S2A indicates that the presence of three different intercalators - EtBr, YOYO-3, and TOTO-3 (each $0.2 \mu \mathrm{M})$ - all caused greater changes to the values of the ratios $E x_{650 / 520}$ relative to that of the control (no intercalator) during the determination of ATP using the 35-Apt-Au NPs (3.0 nM). Of these intercalators, the sensitivity toward ATP was highest for the TOTO-3/Apt-Au NP system. We note that TOTO-3 binds to DNA with greater affinity $\left(K_{\mathrm{a}}=\right.$ ca. $\left.10^{9} \mathrm{M}^{-1}\right)$ than $\mathrm{EtBr}$ $\left(K_{\mathrm{a}}=\right.$ ca. $\left.10^{6} \mathrm{M}^{-1}\right)$ and YOYO-3 $\left(K_{\mathrm{a}}=\right.$ ca. $\left.10^{7} \mathrm{M}^{-1}\right)$ do (Bowen and Woodbury, 2003; Mecklenburg et al., 1997; Joseph et al., 1996).

The G-quadruplex of DNA is stabilized by adding molecular crowding agents such as PEG (Vasilevskaya et al., 1995). Fig. S2B provides a plot of the value of $E x_{650 / 520}$ of the 35-AptAu NP solutions against the concentration of ATP $(0-1.2 \mu \mathrm{M})$ in the probe solutions. The inset to Fig. S2B displays the linear relationship $\left(R^{2}=0.99\right)$ that exists between the value of $E x_{650 / 520}$ for this 35-Apt-Au NP solution and the ATP concentration over the range $20.0-100.0 \mathrm{nM}$, with an LOD of $10.0 \mathrm{nM}(\mathrm{S} / \mathrm{N}=3)$. The improved sensitivity (100-fold) is most likely due to the greater binding between the aptamer and ATP as a result of two effects: PEG's promoting of the structural rigidity of the G-quartet and TOTO-3's stabilizing of the stem structure of the aptamer. Interestingly, the dose-response curve depicted in Fig. S2B possesses two turning points, suggest that ATP might have bound to two different binding sites of the aptamer (Miyoshi et al., 2004).

\subsection{Selectivity and application of 35-Apt-Au NP sensing assays}

Fig. S3A (in supplementary information) displays the changes in the value of Ex650/520 of the 35-Apt-Au NPs (3.0 nM) $30 \mathrm{~min}$ after adding various nucleoside triphosphates $(1.0 \mu \mathrm{M})$. The relative response of the 35-Apt-Au NP solution toward ATP was significantly larger than those toward the other three nucleotides (GTP, CTP, and UTP). The responses of the 35Apt-Au NPs toward adenosine and its other phosphorylated compounds, AMP and ADP, were similar to that toward ATP.

Because adenosine is involved in the regulation of renal hemodynamics, tubular reabsorption, and the release of rennin (Vallon et al., 2006), we employed human-urine samples to test the potential of our Apt-Au NP nanosensor for analyzing adenosine in real biological samples. Because the amount of adenosine in urine is at least two orders of magnitude higher than that of its phosphorylated derivatives (Taniai et al., 2006), the response to extinction changes arose mainly from the presence of adenosine. By applying a standard addition technique (plotting the ratio $E x_{650 / 520}$ against the spiked adenosine concentration over the range $0-40.0 \mathrm{nM}$; see Fig. S3B in supplementary information), we determined that the adenosine concentration in one of the representative urine samples was $3.2( \pm 0.2) \mu \mathrm{M}(n=3)$. The recovery percentages of adenosine over the concentration range 0-40.0 nM were $94.2-98.0 \%$.

\section{Conclusions}

We prepared Apt-Au NPs as selective probes for the analysis of adenosine and its phosphorylated derivatives. The sensing mechanism is based on the increased stability of Apt-Au NPs under high salt conditions while binding with targets. Using the Apt-Au NPs nanosensor, we determined the levels of adenosine in urine samples without the need for pretreatment. This simple, sensitive, specific, and cost-effective approach holds great potential for monitoring the adenosine content in urine samples as a means of diagnosing the presence of renal impairment.

\section{Acknowledgment}

This study was supported by the National Science Council of Taiwan under contract NSC 96-2627-M-002-013 and NSC 96-2627-M-002-014. 


\section{Appendix A. Supplementary data}

Supplementary data associated with this article can be found, in the online version, at doi:10.1016/j.bios.2008.02.008.

\section{References}

Bowen, B.P., Woodbury, N.W., 2003. Photochem. Photobiol. 78 (6), 582-586. Elghanian, R., Storhoff, J.J., Mucic, R.C., Letsinger, R.L., Mirkin, C.A., 1997. Science 277, 1078-1081.

Huang, C.-C., Huang, Y.-F., Cao, Z., Tan, W., Chang, H.-T., 2005. Anal. Chem. 77 (17), 5735-5741.

Huang, Y.-F., Chang, H.-T., 2007. Anal. Chem. 79 (13), 4852-4859.

Huizenga, D.E., Szostak, J.W., 1995. Biochemistry 34 (2), 656-665.

Jhaveri, S.D., Kirby, R., Conrad, R., Maglott, E.J., Bowser, M., Kennedy, R.T., Glick, G., Ellington, A.D., 2000. J. Am. Chem. Soc. 122 (11), 2469-2473.

Joseph, M.J., Taylor, J.C., McGown, L.B., Pitner, J.B., Linn, C.P., 1996. Biospectroscopy 2, 173-183.

Kim, Y., Johnson, R.C., Hupp, J.T., 2001. Nano Lett. 1 (4), 165-167.

Lai, R.Y., Plaxco, K.W., Heeger, A.J., 2007. Anal. Chem. 79 (1), 229-233.

Li, Y.-Y., Zhang, C., Li, B.-S., Zhao, L.-F., Li, X.-B., Yang, W.-J., Xu, S.-Q., 2007. Clin. Chem. 53 (6), 1061-1066.

Lin, C.H., Patel, D.J., 1997. Chem. Biol. 4 (11), 817-832.

Liu, J., Lu, Y., 2006. Angew. Chem. Int. Ed. 45, 90-94.
Mecklenburg, M., Grauers, A., Jönsson, B.R., Weber, A., Danielsson, B., 1997. Anal. Chim. Acta 347, 79-86.

Michaud, M., Jourdan, E., Ravelet, C., Villet, A., Ravel, A., Grosset, C., Peyrin, E., 2004. Anal. Chem. 76 (4), 1015-1020.

Miyoshi, D., Matsumura, S., Nakano, S.-i., Sugimoto, N., 2004. J. Am. Chem. Soc. 126 (1), 165-169.

Nam, J.-M., Park, S.-J., Mirkin, C.A., 2002. J. Am. Chem. Soc. 124 (15), 3820-3821.

Pérez-Ruiz, T., Martínez-Lozano, C., Tomás, V., Martín, J., 2003. Anal. Bioanal. Chem. 377, 189-194.

Shenton, W., Davis, S.A., Mann, S., 1999. Adv. Mater. 11 (6), 449-452.

Storhoff, J.J., Elghanian, R., Mucic, R.C., Mirkin, C.A., Letsinger, R.L., 1998. J. Am. Chem. Soc. 120 (9), 1959-1964.

Taniai, H., Sumi, S., Ito, T., Ueta, A., Ohkubo, Y., Togari, H., 2006. Tohoku J. Exp. Med. 208, 57-63.

Tuerk, C., Gold, L., 1990. Science 249, 505-510.

Vallon, V., Mühlbauer, B., Osswald, H., 2006. Physiol. Rev. 86, 901-940.

Vasilevskaya, V.V., Khokhlov, A.R., Matsuzawa, Y., Yoshikawa, K., 1995. J. Chem. Phys. 102 (16), 6595-6602.

Wang, J., Jiang, Y., Zhou, C., Fang, X., 2005. Anal. Chem. 77 (11), 3542-3546.

Wang, L., Liu, X., Hu, X., Song, S., Fan, C., 2006. Chem. Commun., 3780-3782.

Wu, Z.-S., Guo, M.-M., Zhang, S.-B., Chen, C.-R., Jiang, J.-H., Shen, G.-L., Yu, R.-Q., 2007. Anal. Chem. 79 (7), 2933-2939.

Yoshida, W., Sode, K., Ikebukuro, K., 2006. Anal. Chem. 78 (10), 3296-3303.

Zhao, W., Chiuman, W., Brook, M.A., Li, Y., 2007. ChemBioChem 8, 727-731. 